\title{
PERIODIC SOLUTIONS FOR NONLINEAR DIFFERENTIAL SYSTEMS: THE SECOND ORDER BIFURCATION FUNCTION
}

\author{
ADRIANA BUICĂ ${ }^{1}$, JAUME GINÉ ${ }^{2}$ AND JAUME LLIBRE ${ }^{3}$ \\ ${ }^{1}$ Department of Mathematics, \\ Babeş-Bolyai University, RO-400084 Cluj-Napoca, Romania \\ E-mail: abuica@math.ubbcluj.ro \\ ${ }^{2}$ Departament de Matemàtica, Universitat de Lleida, \\ Av. Jaume II, 69, 25001 Lleida, Catalonia, Spain \\ E-mail: gine@matematica.udl.cat \\ ${ }^{3}$ Departament de Matemàtiques, Universitat Autònoma de Barcelona, \\ 08193 Bellaterra, Barcelona, Catalonia, Spain \\ E-mail: jllibre@mat.uab.cat
}

\begin{abstract}
We are concerned here with the classical problem of Poincaré of persistence of periodic solutions under small perturbations. The main contribution of this work is to give the expression of the second order bifurcation function in more general hypotheses than the ones already existing in the literature. We illustrate our main result constructing a second order bifurcation function for the perturbed symmetric Euler top.
\end{abstract}

\section{IntRoduction}

We are concerned here with the classical problem of Poincaré of persistence of periodic solutions under small perturbations. More precisely, we consider a family of $T$-periodic, sufficiently smooth, $n$-dimensional systems of the form

$$
x^{\prime}(t)=F(t, x, \varepsilon),
$$

depending on a small (perturbation) parameter $\varepsilon$. We assume that there exists some nonempty set $\mathcal{Z}$ whose points are initial values for $T$-periodic solutions of the unperturbed system

$$
x^{\prime}(t)=F(t, x, 0) .
$$

In the following we consider that $\mathcal{Z}$ is the image of some sufficiently smooth $\left(C^{2}\right)$, one-to-one function $\xi: U \rightarrow \mathbb{R}^{n}$, where $U$ is an open subset of $\mathbb{R}^{k}, 1 \leq k \leq n$, such that $D \xi(h)$ has full rank for any $h \in U$. Such a $\mathcal{Z}$ will be called a $T$-period manifold for (2).

Additional hypothesis on $\mathcal{Z}$ is that it is normally nondegenerate (following the terminology of [13]), that means that the linearized system of (2) around each $T$-periodic solution that initiates in $\mathcal{Z}$ has the Floquet multiplier +1 with the geometric multiplicity $k$. This will be explained in detail in Section 3 .

2010 Mathematics Subject Classification. Primary: 34C29, 34C25; Secondary: 58F22.

Key words and phrases. Periodic solution, Lyapunov-Schmidt reduction, period manifold, small parameter, the second order bifurcation function. 
We say that a $T$-periodic solution $\varphi(t)$ of (2) persists in (1) if there exists a $T$-periodic solution $\varphi_{\varepsilon}(t)$ of $(1)$, for small $\varepsilon$ and $\lim _{\varepsilon \rightarrow 0} \varphi_{\varepsilon}(0)=\varphi(0)$.

We say that $f: U \rightarrow \mathbb{R}^{k}$ is a bifurcation function for the problem of persistence in (1) of $T$-periodic solutions of (2) that initiates in $\mathcal{Z}$ if:

(i) for any $\varphi(t)$ with $\varphi(0)=\xi\left(h_{0}\right) \in \mathcal{Z}$ that persists we have that $f\left(h_{0}\right)=0$;

(ii) whenever there exists $h_{0} \in U$ such that $f\left(h_{0}\right)=0$ and the Jacobian determinant det $D f\left(h_{0}\right) \neq 0$, the solution $\varphi(t)$ with $\varphi(0)=\xi\left(h_{0}\right)$ persists.

With this new definition, it is clear that (as it is also well-known) our objective will be to determine the expression of some bifurcation function. Usually this is achieved after a careful study of the Poincaré return map at time $T, z \mapsto x(T, z, \varepsilon)$, whose fixed points are in one-to-one correspondence with the $T$-periodic solutions of $(1)$. Here $x(\cdot, z, \varepsilon)$ is the solution of $(1)$ such that $x(0, z, \varepsilon)=z$. When the expression of the bifurcation function involves only the coefficients of the powers of $\varepsilon$ up to degree $m$ in the Taylor expansion of $x(T, z, \varepsilon)$ around $\varepsilon=0$, we say that the bifurcation function has order $m$.

The main contribution of this work is to give the expression of the second order bifurcation function in the general hypotheses listed above (see Theorem 4). In this way we extend our previous results in [4], where more restrictive hypotheses on $\mathcal{Z}$ were considered. The first order bifurcation function $f_{1}(\alpha)$ of Theorem 4 goes back to Malkin [12] and Roseau [14], see also the book of Françoise [9]. For a shorter proof of this first result see [2]. The first order bifurcation function was also computed in a nonsmooth setting in [6]. The second order bifurcation function $f_{2}(\alpha)$ of Theorem 4 was given in [4] but under assumptions more restrictive than the ones that we suppose here. For a short history of this intensively studied problem we refer also to our paper [4]. Other references that are closely related to this work are $[5,6,7,8,10,11,13]$.

It is important also to notice that we present two proofs for our main result. The second proof reveals the simple but surprising theoretical fact that there exists a linear change of variables that transforms a system satisfying the hypotheses listed above in a system satisfying the hypotheses of the main result of [4]. The new result presented here (Theorem 4) is useful in some applications where the main result provided in [4] is not applicable. Moreover, we enrich our previous result proving the first property in the definition of the bifurcation function given above, i.e. we also show that for any $T$-periodic solution that persists, there exists a zero of the bifurcation function.

We illustrate our main result constructing a second order bifurcation function for the perturbed symmetric Euler top. The first order bifurcation function for this system was found in [3].

At the end of the Introduction we list some notations used in this paper. We denote the projection onto the first $k$ coordinates by $\pi: \mathbb{R}^{k} \times \mathbb{R}^{n-k} \rightarrow \mathbb{R}^{k}$ and the one onto the last $(n-k)$ coordinates by $\pi^{\perp}: \mathbb{R}^{k} \times \mathbb{R}^{n-k} \rightarrow \mathbb{R}^{n-k}$. For the $n$-dimensional function $g$ of $n$ variables $z=(\alpha, \beta) \in \mathbb{R}^{k} \times \mathbb{R}^{n-k}$, we denote by $D g$ or $D_{z} g$ (in the case that it depends also on other variables) the Jacobian matrix of $g$, by $D(\pi g)$ the $k \times n$ Jacobian matrix of the $k$-dimensional function $\pi g$, by $D_{\beta}(\pi g)$ the $k \times(n-k)$ Jacobian matrix of $\beta \in \mathbb{R}^{n-k} \mapsto \pi g(\alpha, \beta) \in \mathbb{R}^{k}$. We also denote by $\frac{\partial}{\partial z_{i}}(D g)$ the matrix of the same dimension as $D g$ whose entries are the first order partial derivatives with respect to the component $z_{i}$ of $z$, of the entries of $D g$. 
For a matrix $M$ with $n$ lines, we denote by $\pi M$ the matrix formed by the first $k$ lines of $M$, and by $\pi^{\perp} M$ the matrix formed by the last $(n-k)$ lines of $M$. For any matrix (or vector) $M$ we denote by $M^{*}$ its transpose.

\section{LYAPUNOV-SCHMidT REDUCTION THEOREM FOR FINITE DIMENSIONAL FUNCTIONS}

In this section we consider the problem of persistence of zeros of finite dimensional maps under small perturbations. We start by presenting a terminology for this problem which is analogous with the one gave in the Introduction for the problem of persistence of periodic solutions. We consider a family of $n$-dimensional, sufficiently smooth maps $(z, \varepsilon) \mapsto g(z, \varepsilon)$ depending on the small perturbation parameter $\varepsilon$. We assume that there exists some nonempty set $\mathcal{Z}$ whose points are zeros of $z \mapsto g(z, 0)$. We consider that $\mathcal{Z}$ is the image of some sufficiently smooth $\left(C^{2}\right)$, one-to-one function $\xi: U \rightarrow \mathbb{R}^{n}$, where $U$ is an open subset of $\mathbb{R}^{k}, 1 \leq k \leq n$, such that $D \xi(h)$ has full rank for any $h \in U$. Such a $\mathcal{Z}$ will be called zero-manifold for the map $z \mapsto g(z, 0)$. We say that the zero-manifold of $g(\cdot, 0)$ is normally nondegenerate if the Jacobian matrix $D_{z} g(\cdot, \varepsilon)(\xi(h))$ has rank $n-k$.

We say that a zero $z_{0}$ of $g(\cdot, 0)$ persists as zero of $g(\cdot, \varepsilon)$ if there exists $z_{\varepsilon}$, for small $\varepsilon$, such that $g\left(z_{\varepsilon}, \varepsilon\right)=0$ and $\lim _{\varepsilon \rightarrow 0} z_{\varepsilon}=z_{0}$.

We say that $f: U \rightarrow \mathbb{R}^{k}$ is a bifurcation function for the problem of persistence of zeros in the family $g(\cdot, \varepsilon)$ if:

(i) for any $z_{0}=\xi\left(h_{0}\right) \in \mathcal{Z}$ that persists we have $f\left(h_{0}\right)=0$;

(ii) whenever there exists $h_{0} \in U$ such that $f\left(h_{0}\right)=0$ and the Jacobian determinant det $D f\left(h_{0}\right) \neq 0$, the zero $z_{0}=\xi\left(h_{0}\right)$ of $g(\cdot, 0)$ persists.

When the expression of the bifurcation function involves only the coefficients of the powers of $\varepsilon$ up to degree $m$ in the Taylor expansion of $g(z, \varepsilon)$ around $\varepsilon=0$, we say that the bifurcation function has order $m$. For example, if we write

$$
g(z, \varepsilon)=g_{0}(z)+\varepsilon g_{1}(z)+\varepsilon^{2} g_{2}(z)+O\left(\varepsilon^{3}\right),
$$

the expression of a second order bifurcation function will be constructed using $g_{0}$, $g_{1}$ and $g_{2}$.

The main result of this section will be proved using the following Theorem from our previous paper [4]. Since in [4] only the second property in the definition of the bifurcation function is proved, we complete here with the main ideas of the proof of the first property.

Theorem 1. Let $g_{0}, g_{1}, g_{2}: D \rightarrow \mathbb{R}^{n}$ and $\beta: V \rightarrow \mathbb{R}^{n-k}$ be $C^{2}$ functions, where $D$ is an open subset of $\mathbb{R}^{n}$ and $V$ is an open and bounded subset of $\mathbb{R}^{k}$. Denote $\zeta(\alpha)=\left(\begin{array}{l}\alpha \\ \beta(\alpha)\end{array}\right)$ for any $\alpha \in V$. We assume that

(i) $\zeta(V) \subset D$ is a zero-manifold for $g_{0}$,

(ii) the Jacobian matrix $G_{\alpha}=D g_{0}(\zeta(\alpha))$ has in the right up corner the null $k \times(n-k)$ matrix, while in the right down corner has the $(n-k) \times(n-k)$ matrix $\Delta_{\alpha}$, with $\operatorname{det}\left(\Delta_{\alpha}\right) \neq 0$.

We consider the functions $f_{1}: V \rightarrow \mathbb{R}^{k}$ defined by

$$
f_{1}(\alpha)=\pi g_{1}(\zeta(\alpha))
$$


and $f_{2}: V \rightarrow \mathbb{R}^{k}$ defined by

$$
\begin{aligned}
f_{2}(\alpha)= & 2\left(\pi g_{2}\right)(\zeta(\alpha))+2 D_{\beta}\left(\pi g_{1}\right)(\zeta(\alpha)) \gamma(\alpha) \\
& +\sum_{i=1}^{n-k} \gamma_{i}(\alpha) \frac{\partial}{\partial z_{k+i}} D_{\beta}\left(\pi g_{0}\right)(\zeta(\alpha)) \gamma(\alpha),
\end{aligned}
$$

where

$$
\gamma(\alpha)=-\Delta_{\alpha}^{-1}\left(\pi^{\perp} g_{1}\right)(\zeta(\alpha))=\left(\gamma_{1}(\alpha), \ldots, \gamma_{n-k}(\alpha)\right)^{*} \in \mathbb{R}^{n-k} .
$$

Then $f_{1}$ is a first order bifurcation function and, when $f_{1}(\alpha) \equiv 0, f_{2}$ is a second order bifurcation function for the problem of persistence of zeros in the family $g(\cdot, \varepsilon)$.

Proof. We present first some useful facts from the proof given in [4].

For each $\alpha \in V$ and $|\varepsilon|$ sufficiently small, there exists a unique $\bar{\beta}(\alpha, \varepsilon)$ such that

$$
\bar{\beta}(\alpha, 0)=\beta(\alpha) \quad \text { and } \quad \pi^{\perp} g(\alpha, \bar{\beta}(\alpha, \varepsilon), \varepsilon)=0 .
$$

Moreover, we have

$$
\delta(\alpha, \varepsilon):=\pi g(\alpha, \bar{\beta}(\alpha, \varepsilon), \varepsilon)=\varepsilon f_{1}(\alpha)+\frac{\varepsilon^{2}}{2} f_{2}(\alpha)+O\left(\varepsilon^{3}\right) .
$$

In order to prove the first property from the definition of the bifurcation function, we fix some $z_{0}=\zeta\left(\alpha_{0}\right)=\left(\alpha_{0}, \beta\left(\alpha_{0}\right)^{*}\right.$ a zero that persists in the family $g(z, \varepsilon)$. Hence there exists $z_{\varepsilon}$ such that $g\left(z_{\varepsilon}, \varepsilon\right) \equiv 0$ and $z_{\varepsilon} \rightarrow z_{0}$ as $\varepsilon \rightarrow 0$. If we denote $\alpha_{\varepsilon}=\pi z_{\varepsilon}$ and $\beta_{\varepsilon}=\pi^{\perp} z_{\varepsilon}$, we can also write that $\pi^{\perp} g\left(\alpha_{\varepsilon}, \beta_{\varepsilon}, \varepsilon\right) \equiv 0$. From the uniqueness of $\bar{\beta}$ we deduce that $\beta_{\varepsilon}=\bar{\beta}\left(\alpha_{\varepsilon}, \varepsilon\right)$. Then $\delta\left(\alpha_{\varepsilon}, \varepsilon\right)=\pi g\left(\alpha_{\varepsilon}, \beta_{\varepsilon}, \varepsilon\right) \equiv 0$. Hence $\delta\left(\alpha_{\varepsilon}, \varepsilon\right)=\varepsilon f_{1}\left(\alpha_{\varepsilon}\right)+\frac{\varepsilon^{2}}{2} f_{2}\left(\alpha_{\varepsilon}\right)+O\left(\varepsilon^{3}\right) \equiv 0$ and we remind that $\alpha_{\varepsilon} \rightarrow \alpha_{0}$ as $\varepsilon \rightarrow 0$. Passing to the limit as $\varepsilon \rightarrow 0$ in $\frac{1}{\varepsilon} \delta\left(\alpha_{\varepsilon}, \varepsilon\right) \equiv 0$ we obtain that $f_{1}\left(\alpha_{0}\right)=0$. If $f_{1}(\alpha) \equiv 0$, passing to the limit as $\varepsilon \rightarrow 0$ in $\frac{1}{\varepsilon^{2}} \delta\left(\alpha_{\varepsilon}, \varepsilon\right) \equiv 0$ we obtain that $f_{2}\left(\alpha_{0}\right)=0$.

The main result of this section is the next one which essentially generalizes Theorem 1 given in [4].

Theorem 2. Let $g_{0}, g_{1}, g_{2}: D \rightarrow \mathbb{R}^{n}$ and $\xi: U \rightarrow \mathbb{R}^{n}$ be $C^{2}$ functions, where $D$ is an open subset of $\mathbb{R}^{n}, U$ is an open subset of $\mathbb{R}^{k}$. We assume that for each $h \in U$ we have

(i) $\xi(U) \subset D$ is a normally nondegenerate zero-manifold for $g_{0}$,

(ii) the first $k$ lines of $D g_{0}(\xi(h))$ are null vectors (hence there exists some $n \times$ $(n-k)$ matrix $S$ such that the $(n-k) \times(n-k)$ matrix $D\left(\pi^{\perp} g_{0}\right)(\xi(h)) S$ is invertible). Assume that $S$ is constant with respect to $h \in U$.

We consider the function $f_{1}: U \rightarrow \mathbb{R}^{k}$ defined by

$$
f_{1}(h)=\pi g_{1}(\xi(h))
$$

and $f_{2}: V \rightarrow \mathbb{R}^{k}$ defined by

$$
\begin{aligned}
f_{2}(h)= & 2\left(\pi g_{2}\right)(\xi(h))+2 D\left(\pi g_{1}\right)(\xi(h)) S \gamma(h) \\
& +\sum_{i=1}^{n-k} \gamma_{i}(h)\left[\frac{\partial}{\partial z_{k+i}} D\left(\pi g_{0}\right)\right](\xi(h)) S \gamma(h),
\end{aligned}
$$


where

$$
\gamma(h)=-\left[D\left(\pi^{\perp} g_{0}\right)(\xi(h)) S\right]^{-1}\left(\pi^{\perp} g_{1}\right)(\xi(h))=\left(\gamma_{1}(h), \ldots, \gamma_{n-k}(h)\right)^{*} \in \mathbb{R}^{n-k} .
$$

Then $f_{1}$ is a first order bifurcation function and, when $f_{1}(\alpha) \equiv 0, f_{2}$ is a second order bifurcation function for the problem of persistence of zeros in the family $g(\cdot, \varepsilon)$.

Proof. Let $h \in U$ be arbitrary. There exists some $n \times n$ invertible matrix $\tilde{S}$ whose last $(n-k)$ columns are the columns of the matrix $S$ chosen in the hypothesis (ii), since the columns of $S$ must be linearly independent. Then $D g_{0}(\xi(h)) \tilde{S}$ has in its first $k$ lines null entries, and in the right down corner an $(n-k) \times(n-k)$ invertible matrix, which means that

$$
D g_{0}(\xi(h)) \tilde{S} \text { has the same structure as } G_{\alpha} \text { in (ii) of Theorem } 1 .
$$

Our goal is to apply Theorem 1 to $\tilde{g}: D \times\left(-\varepsilon_{0}, \varepsilon_{0}\right) \rightarrow \mathbb{R}^{n}$ defined by

$$
\tilde{g}(z, \varepsilon)=g(\tilde{S} z, \varepsilon)
$$

and $\beta: V \in \mathbb{R}^{n-k}$ defined such that for any $\alpha \in V$

$$
\xi(\tilde{h}(\alpha))=\tilde{S}\left(\begin{array}{c}
\alpha \\
\beta(\alpha)
\end{array}\right),
$$

with some invertible function $\tilde{h}: V \rightarrow U$ whose existence will be proved later on. Indeed, if we denote

$$
\tilde{g}_{i}(z)=g_{i}(\tilde{S} z), \quad i \in\{0,1,2\}
$$

one can write

$$
\tilde{g}(z, \varepsilon)=\tilde{g}_{0}(z)+\varepsilon \tilde{g}_{1}(z)+\varepsilon^{2} \tilde{g}_{2}(z)+O\left(\varepsilon^{3}\right) .
$$

Moreover one can see that $D \tilde{g}_{0}(z)=D g_{0}(\tilde{S} z) \tilde{S}$ and, in particular, using (8) and the notation of Theorem 1 (i), for any $\alpha \in V$,

$$
\tilde{g}_{0}(\zeta(\alpha))=g_{0}\left(\xi(\tilde{h}(\alpha)), \quad D \tilde{g}_{0}(\zeta(\alpha))=D g_{0}(\xi(\tilde{h}(\alpha)) \tilde{S} .\right.
$$

Now from our hypothesis (i) of Theorem 2, relations (7) and (9) the hypotheses (i) and (ii) of Theorem 1 are fulfilled.

It remains to prove that equation (8) is valid. Notice that it can be equivalently written as

$$
\alpha=\pi(\tilde{S})^{-1} \xi(\tilde{h}(\alpha)), \quad \beta(\alpha)=\pi^{\perp}(\tilde{S})^{-1} \xi(\tilde{h}(\alpha)) .
$$

We must to prove the existence of $\tilde{h}$ satisfying the first of the two previous equations, the second one being the definition of $\beta$. By hypothesis, we have that the function defined by $h \in U \mapsto \pi(\tilde{S})^{-1} \xi(h)$ is one-to-one and we claim that its image is an open subset of $\mathbb{R}^{k}$, which will be denoted by $V$. Take $\tilde{h}: V \rightarrow U$ be its inverse. In the following we prove this claim, showing that the derivative of function defined by $h \in U \mapsto \pi(\tilde{S})^{-1} \xi(h)$, in any $h$, is a nonsingular matrix. Taking the derivative with respect to $h$ in $g_{0}(\xi(h)) \equiv 0$ we obtain $D g_{0}(\xi(h)) D \xi(h) \equiv 0_{n \times k}$ and, further,

$$
D g_{0}(\xi(h)) \tilde{S}(\tilde{S})^{-1} D \xi(h) \equiv 0_{n \times k} .
$$

Since $D g_{0}(\xi(h)) \tilde{S}$ has in its right down corner an $(n-k) \times(n-k)$ invertible matrix, from (10) we obtain that there exists some $(n-k) \times k$ matrix $A(h)$ such that

$$
\pi^{\perp}(\tilde{S})^{-1} D \xi(h)=A(h) \pi(\tilde{S})^{-1} D \xi(h) .
$$


From (i), $D \xi(h)$ has rank $k$, hence also $(\tilde{S})^{-1} D \xi(h)$ has rank $k$ and, using (11), we further deduce that the $k \times k$ matrix $\pi(\tilde{S})^{-1} D \xi(h)$ is nonsingular. So the claim is proved.

We are now concerned with the expressions of the bifurcation functions. The expression of the first order bifurcation function corresponding to $\tilde{g}(z, \varepsilon)$ given in Theorem 1 is

$$
\tilde{f}_{1}(\alpha)=\pi \tilde{g}_{1}(\alpha, \beta(\alpha))=\pi g_{1}(\xi(\tilde{h}(\alpha))) .
$$

Since $\alpha \mapsto \tilde{h}(\alpha)$ is a homeomorphism, we can simply consider the first order bifurcation function given by (5).

Using the same ideas and since for any $z \in D$,

$$
D_{\beta}\left(\pi \tilde{g}_{i}\right)(z)=D\left(\pi g_{i}\right)(\tilde{S} z) S, \text { for } i=1,2,
$$

and $\Delta_{\alpha}=D_{\beta}\left(\pi^{\perp} \tilde{g}_{0}\right)(z)=D\left(\pi^{\perp} g_{0}\right)(\tilde{S} z) S$, one can find the expression of the second order bifurcation function (6).

\section{MAIN RESULT}

We start this section with the following lemma on linear periodic differential systems.

Lemma 3. For each $\xi \in \mathbb{R}^{n}$ we consider the $T$-periodic $n$-dimensional linear differential system

$$
y^{\prime}=P(t, \xi) y
$$

where $P$ is continuous in $t$ and of class $C^{2}$ in $\xi$. Assume that system (12) has the Floquet multiplier +1 with the geometric multiplicity equal to $k$. Then there exists a fundamental matrix solution $Y(t, \xi)$ of system (12) with $C^{2}$-dependence on $\xi$ such that

$$
Y(0, \xi)^{-1}-Y(T, \xi)^{-1}
$$

has rank $(n-k)$ and its first $k$ lines are null.

Moreover, there exists some $n \times(n-k)$ matrix $S(\xi)$ such that the $(n-k) \times(n-k)$ matrix

is invertible.

$$
\pi^{\perp}\left(Y(0, \xi)^{-1}-Y(T, \xi)^{-1}\right) S(\xi)
$$

Proof. Let $Y(t, \xi)$ be some arbitrary fundamental matrix solution of (12). By definition, +1 is a Floquet multiplier of geometric multiplicity $k$ when the kernel of $Y(T, \xi) Y(0, \xi)^{-1}-I_{n}$ has dimension $k$ (here $I_{n}$ is the $n \times n$ identity matrix). From here, the kernel of $Y(0, \xi)^{-1}-Y(T, \xi)^{-1}$ has also dimension $k$ and rank $(n-k)$. The $k$ linearly independent vectors from this kernel are initial values for $k$ linearly independent $T$-periodic solutions of (12). It follows that the adjoint system

$$
y^{\prime}=-[P(t, \xi)]^{*} y,
$$

has $k$ linearly independent $T$-periodic solutions. We denote them by $u_{i}(t, \xi)$ for $i=1, \ldots, k$. Choose now a fundamental matrix solution $U(t, \xi)$ of the adjoint system (13) whose first $k$ columns are the $T$-periodic solutions $u_{i}(t, \xi)$ for $i=$ $1, \ldots, k$. Then choose $Y(t, \xi)$ the fundamental matrix solution of (12) such that $Y(t, \xi)^{-1}=U(t, \xi)^{*}$. It follows that the first $k$ lines of $Y(t, \xi)^{-1}$ are $u_{i}(t, \xi)$ for $i=1, \ldots, k$, hence the first $k$ lines of $Y(0, \xi)^{-1}-Y(T, \xi)^{-1}$ are null. In order to assure the $C^{2}$-dependence on $\xi$ of $Y(t, \xi)$ for any $t$, it is sufficient to assure this 
for $t=0$. This will follow showing that one can choose $k$ linearly independent (as vectors in $\left.\mathbb{R}^{n}\right) u_{i}(0, \xi)$ for $i=1, \ldots, k$, initial conditions for $T$-periodic solutions of the adjoint system (13). This is indeed valid since these vectors must be a base in the kernel of the displacement map of the adjoint system (13), and the displacement map has $C^{2}$-dependence with respect to the parameter $\xi$.

One can choose the $(n-k)$ columns of the matrix $S(\xi)$ to be a base in the range of $Y(0, \xi)^{-1}-Y(T, \xi)^{-1}$.

Lemma 3 will be applied to the variational equation of the unperturbed system (2) associated to its solution $x(t, z, 0)$,

$$
y^{\prime}=D_{x} F_{0}(t, x(t, z, 0)) y
$$

where $F_{0}(t, x)=F(t, x, 0)$. In fact we will need more notations from the Taylor expansion of $F(t, x, \varepsilon)$ around $\varepsilon=0$,

$$
F(t, x, \varepsilon)=F_{0}(t, x)+\varepsilon F_{1}(t, x)+\varepsilon^{2} F_{2}(t, x)+O\left(\varepsilon^{3}\right) .
$$

The main result of this paper is the following:

Theorem 4. Let $F_{0}, F_{1}, F_{2}: \mathbb{R} \times \Omega \rightarrow \mathbb{R}^{n}$ and $\xi: U \rightarrow \mathbb{R}^{n}$ be $C^{2}$ functions, where $\Omega$ is an open subset of $\mathbb{R}^{n}$ and $U$ is an open subset of $\mathbb{R}^{k}$. We assume that $\xi(U) \subset D$ is a normally nondegenerate $T$-periodic manifold for system (2).

Let $Y(t, \xi(h))$ be the fundamental matrix solution of (14) with $z=\xi(h)$ and let $S(\xi(h))$ be as in Lemma 3 applied to the linear differential system (14). Assume that $S$ is constant with respect to $h \in U$.

We consider the functions $f_{1}: U \rightarrow \mathbb{R}^{k}$ defined by

$$
f_{1}(h)=\pi g_{1}(\xi(h))
$$

and $f_{2}: U \rightarrow \mathbb{R}^{k}$ defined by

$$
f_{2}(h)=2\left(\pi g_{2}\right)(\xi(h))+2 D\left(\pi g_{1}\right)(\xi(h)) S \gamma(h)+\sum_{i=1}^{n-k} \gamma_{i}(h)\left[\frac{\partial}{\partial z_{k+i}} D\left(\pi g_{0}\right)\right](\xi(h)) S \gamma(h),
$$

where

and

$$
\gamma(h)=-\left[D\left(\pi^{\perp} g_{0}\right)(\xi(h)) S\right]^{-1}\left(\pi^{\perp} g_{1}\right)(\xi(h)) \in \mathbb{R}^{n-k},
$$

with

$$
\begin{aligned}
& g_{0}(z)=Y(T, z)^{-1}(x(T, z, 0)-z), \\
& g_{1}(z)=\int_{0}^{T} Y(t, z)^{-1} F_{1}(t, x(t, z, 0)) d t, \\
& g_{2}(z)=\frac{1}{2} \int_{0}^{T} Y(t, z)^{-1} F_{*}(t, x(t, z, 0)) d t,
\end{aligned}
$$

$$
\begin{aligned}
F_{*} & =2 F_{2}+2\left(D_{x} F_{1}\right) \frac{\partial x}{\partial \varepsilon}+\sum_{i=1}^{n} \frac{\partial x_{i}}{\partial \varepsilon} \frac{\partial}{\partial x_{i}}\left(D_{x} F_{0}\right) \frac{\partial x}{\partial \varepsilon} \\
\frac{\partial x}{\partial \varepsilon}(t, z, 0) & =Y(t, z) \int_{0}^{t} Y(s, z)^{-1} F_{1}(s, x(s, z, 0)) d s=\left(\frac{\partial x_{1}}{\partial \varepsilon}, \ldots, \frac{\partial x_{n}}{\partial \varepsilon}\right)^{*} .
\end{aligned}
$$

Then $f_{1}$ is a first order bifurcation function and, when $f_{1}(\alpha) \equiv 0, f_{2}$ is a second order bifurcation function for the problem of persistence in (1) of T-periodic solutions of (2) that initiates in $\mathcal{Z}$. 
Remark 5. Let $u_{i}(\cdot, h)$ for $i=1, \ldots, k$ be linearly independent $T$-periodic solutions of the adjoint linear variational system $y^{\prime}=-\left[D_{x} F_{0}(t, x(t, z, 0))\right]^{*} y$ and let $Y(t, \xi(h))$ be as in Theorem 4. Then, from the proof of Lemma 3 we have that the first $k$ lines of $Y(t, \xi(h))$ are $u_{i}(\cdot, h)$ for $i=1, \ldots, k$. Hence

$$
\pi g_{1}(\xi(h))=\int_{0}^{T}\left(\begin{array}{l}
u_{1}(t, h) \\
\ldots \\
u_{k}(t, h)
\end{array}\right) F_{1}(t, x(t, \xi(h), 0)) d t
$$

Written in this form, $f_{1}(h)=\pi g_{1}(\xi(h))$ is named the Malkin bifurcation function.

Note that in order to find the expression of the second order bifurcation function $f_{2}$ it is necessary to know all the entries of $Y(t, \xi(h))$.

Remark 6. For calculating $f_{2}$ we need to know the derivatives of $g_{1}$ and $g_{2}$. It might be useful to know that $D_{z} x(t, z, 0)$ is the principal fundamental matrix of (14).

Corollary 7 (The isochronous case). We assume that there exists an open set $U \subset D$ such that for each $z \in U, x(\cdot, z, 0)$ is T-periodic, that is the hypotheses of the above theorem are fulfilled for $k=n$. In this case $g_{0} \equiv 0$ and the bifurcation functions have simpler expressions $f_{1}(z)=g_{1}(z)$ and $f_{2}(z)=2 g_{2}(z)$, where $g_{1}$ and $g_{2}$ are calculated according to the formulas of Theorem 4 .

Remark 8. We present two proofs for Theorem 4. In the first proof we apply the Lyapunov-Schmidt reduction of Theorem 2 to the Poincaré return map. In the second proof we show that there exists a linear change of variables that transforms a system which is in the conditions of Theorem 4 into a system that fulfills the hypotheses of the main result in [4] (Theorem 9 below).

First proof of Theorem 4. For $z \in \Omega$ we denote by $x(\cdot, z, \varepsilon):\left[0, t_{(z, \varepsilon)}\right) \rightarrow \mathbb{R}^{n}$ the solution of (1) with $x(0, z, \varepsilon)=z$. From Theorem 8.3 of [1] we deduce that, whenever $t_{\left(z_{0}, 0\right)}>T$ for some $z_{0} \in \Omega$ there exists a neighborhood of $\left(z_{0}, 0\right)$ in $\Omega \times\left(-\varepsilon_{f}, \varepsilon_{f}\right)$ such that, for all $(z, \varepsilon)$ in this neighborhood, $t_{(z, \varepsilon)}>T$. Under this assumption there exists an open subset $D$ of $\Omega$ and a sufficiently small $\varepsilon_{0}>0$ such that, for all $(z, \varepsilon) \in D \times\left(-\varepsilon_{0}, \varepsilon_{0}\right)$, the solution $x(\cdot, z, \varepsilon)$ is defined on the interval $[0, T]$. Hence, the Poincaré return map $x(T, z, \varepsilon)$ is well-defined for each $z \in D$ and $\varepsilon \in\left(-\varepsilon_{0}, \varepsilon_{0}\right)$. We remind that the $T$-periodic solutions of (1) that initiates in $D$ are in one-to-one correspondence with the fixed points of $x(T, \cdot, \varepsilon)$.

As in the proof of Theorem 1 in [4], we consider

$$
g(z, \varepsilon)=Y(T, z)^{-1}(x(T, z, \varepsilon)-z)
$$

and note that its zeros are in one-to-one correspondence with the $T$-periodic solutions of (1). To this function we shall apply Theorem 2. Note that $\xi: U \rightarrow \mathbb{R}^{n}$ satisfies the assumptions of Theorem 2. We have to identify the functions $g_{0}, g_{1}$ and $g_{2}$ and to prove that $g_{0}$ satisfies the hypotheses of Theorem 2. Of course, $g_{0}(z)=g(z, 0)=Y(T, z)^{-1}(x(T, z, 0)-z)$ and, since $\xi(h)$ is the initial value of some $T$-periodic solution of $(2)$, we have that $g_{0}(\xi(h))=0$ for any $h \in U$. We claim that

$$
D g_{0}(\xi(h))=Y(0, \xi(h))^{-1}-Y(T, \xi(h))^{-1} .
$$

Then, using the hypothesis that $\xi(U) \subset D$ is a normally nondegenerate $T$-periodic manifold for system (2) (mainly that system (14) for $z=\xi(h)$ has the Floquet 
multiplier +1 with the geometric multiplicity equal to $k$ ), by Lemma 3 follows that $D g_{0}(\xi(h))$ satisfies hypothesis (ii) of Theorem 2 .

In order to prove the claim we need to know $\left(D_{z} x\right)(t, z, 0)$. Since it is the matrix solution of (14) with $\left(D_{z} x\right)(0, z, 0)=I_{n}$, we have that $\left(D_{z} x\right)(t, z, 0)=$ $Y(t, z) Y(0, z)^{-1}$. Moreover,

$$
D_{z}(x(T, z, 0)-z)=D_{z} x(T, z, 0)-I_{n}=Y(T, z) Y(0, z)^{-1}-I_{n}
$$

and

$D g_{0}(z)=Y(0, z)^{-1}-Y(T, z)^{-1}+\left(\frac{\partial}{\partial z_{1}} Y(T, z)^{-1} f(z, 0), \ldots, \frac{\partial}{\partial z_{n}} Y(T, z)^{-1} f(z, 0)\right)$, that, for $z=\xi(h)$ reduces to (17).

In short, all the assumptions hypotheses of Theorem 2 are fulfilled. In order to find the expressions of the bifurcation functions it remains only to find the expressions of $g_{1}$ and $g_{2}$. Note that this is already done in the proof of Theorem 1 of [4], but for completeness we present here the main ideas. We have

$$
g_{1}(z)=\frac{\partial g}{\partial \varepsilon}(z, 0)=Y(T, z)^{-1} \frac{\partial x}{\partial \varepsilon}(T, z, 0) .
$$

Taking the derivative with respect to $\varepsilon$ in the relations

$$
\begin{aligned}
& x^{\prime}(t, z, \varepsilon)=F_{0}(t, x(t, z, \varepsilon))+\varepsilon F_{1}(t, x(t, z, \varepsilon))+\varepsilon^{2} F_{2}(t, x(t, z, \varepsilon))+O\left(\varepsilon^{3}\right), \\
& x(0, z, \varepsilon)=z,
\end{aligned}
$$

one can see that the function $(\partial x / \partial \varepsilon)(\cdot, z, 0)$ is the unique solution of the initial value problem

$$
y^{\prime}=D_{x} F_{0}(t, x(t, z, 0)) y+F_{1}(t, x(t, z, 0)), \quad y(0)=0 .
$$

Hence

$$
\frac{\partial x}{\partial \varepsilon}(t, z, 0)=Y(t, z) \int_{0}^{t} Y(s, z)^{-1} F_{1}(s, x(s, z, 0)) d s .
$$

Then we have

$$
g_{1}(z)=\frac{\partial g}{\partial \varepsilon}(z, 0)=\int_{0}^{T} Y(s, z)^{-1} F_{1}(s, x(s, z, 0)) d s .
$$

Taking the second order derivative with respect to $\varepsilon$ in the relations (18), we can see that the function $\left(\partial^{2} x / \partial \varepsilon^{2}\right)(\cdot, z, 0)$ is the unique solution of the initial value problem

$$
y^{\prime}=D_{x} F_{0}(t, x(t, z, 0)) y+F_{*}(t, x(t, z, 0)), \quad y(0)=0,
$$

where the expression of $F_{*}$ is given in the statement of the theorem. Hence

$$
\frac{\partial^{2} x}{\partial \varepsilon^{2}}(t, z, 0)=Y(t, z) \int_{0}^{t} Y(s, z)^{-1} F_{*}(s, x(s, z, 0)) d s .
$$

Therefore we have

$$
g_{2}(z)=\frac{1}{2} \frac{\partial^{2} g}{\partial \varepsilon^{2}}(z, 0)=\frac{1}{2} \int_{0}^{T} Y(s, z)^{-1} F_{*}(s, x(s, z, 0)) d s .
$$

We state now the main result in [4] which will be used for a second proof of Theorem 4. 
Theorem 9. Let $F_{0}, F_{1}, F_{2}: \mathbb{R} \times \Omega \rightarrow \mathbb{R}^{n}$ and $\beta: V \rightarrow \mathbb{R}^{n-k}$ be $C^{2}$ functions, where $\Omega$ is an open subset of $\mathbb{R}^{n}$ and $V$ is an open subset of $\mathbb{R}^{k}$. Denote $\zeta(\alpha)=\left(\begin{array}{l}\alpha \\ \beta(\alpha)\end{array}\right)$ for any $\alpha \in V$. We assume that

(i) $\zeta(V) \subset D$ is a T-period manifold of (2);

(ii) for each $\alpha \in V$, there exists a fundamental matrix solution $Y_{\alpha}(t)=Y(t, \zeta(\alpha))$ of (14) such that the matrix $Y_{\alpha}(0)^{-1}-Y_{\alpha}(T)^{-1}$ has in the right up corner the null $k \times(n-k)$ matrix, while in the right down corner has the $(n-k) \times(n-k)$ matrix $\Delta_{\alpha}$, with $\operatorname{det}\left(\Delta_{\alpha}\right) \neq 0$.

We consider the functions $f_{1}: V \rightarrow \mathbb{R}^{k}$ defined by

$$
f_{1}(\alpha)=\pi\left(\int_{0}^{T} Y(t, \zeta(\alpha))^{-1} F_{1}(t, x(t, \zeta(\alpha), 0)) d t\right)
$$

and $f_{2}: V \rightarrow \mathbb{R}^{k}$ defined by

$$
f_{2}(\alpha)=2 \pi g_{2}(\alpha)+2\left(D_{\beta}\left(\pi g_{1}\right)(\zeta(\alpha))\right) \gamma(\alpha)+\sum_{i=1}^{n-k} \gamma_{i}(\alpha) \frac{\partial}{\partial \beta_{i}}\left(D_{\beta}\left(\pi g_{0}\right)(\zeta(\alpha))\right) \gamma(\alpha)
$$

where

and

$$
\gamma(\alpha)=-\Delta_{\alpha}^{-1}\left(\pi^{\perp} g_{1}\right)(\zeta(\alpha))=\left(\gamma_{1}(\alpha), \ldots, \gamma_{n-k}(\alpha)\right)^{*} \in \mathbb{R}^{n-k}
$$

$$
\begin{aligned}
& g_{0}(z)=Y(T, z)^{-1}(x(T, z, 0)-z), \\
& g_{1}(z)=\int_{0}^{T} Y(t, z)^{-1} F_{1}(t, x(t, z, 0)) d t, \\
& g_{2}(z)=\frac{1}{2} \int_{0}^{T} Y(t, z)^{-1} F_{*}(t, x(t, z, 0)) d t,
\end{aligned}
$$

with

$$
\begin{aligned}
F_{*} & =2 F_{2}+2\left(D_{x} F_{1}\right) \frac{\partial x}{\partial \varepsilon}+\sum_{i=1}^{n} \frac{\partial x_{i}}{\partial \varepsilon} \frac{\partial}{\partial x_{i}}\left(D_{x} F_{0}\right) \frac{\partial x}{\partial \varepsilon} \\
\frac{\partial x}{\partial \varepsilon}(t, z, 0) & =Y(t, z) \int_{0}^{t} Y(s, z)^{-1} F_{1}(s, x(s, z, 0)) d s=\left(\frac{\partial x_{1}}{\partial \varepsilon}, \ldots, \frac{\partial x_{n}}{\partial \varepsilon}\right)^{*} .
\end{aligned}
$$

Then $f_{1}$ is a first order bifurcation function and, when $f_{1}(\alpha) \equiv 0, f_{2}$ is a second order bifurcation function for the problem of persistence in (1) of T-periodic solutions of (2) that initiates in $\zeta(V)$.

Second proof of Theorem 4. There exists some $n \times n$ invertible matrix $\tilde{S}$ whose last $(n-k)$ columns are the columns of $S$ because these must be linearly independent. We will prove that, by the linear change of variable

$$
x=\tilde{S} \tilde{x}
$$

system (2) is transformed into a system that satisfies the hypotheses of Theorem 9. As in the proof of Theorem 2 we define $\beta: V \rightarrow \mathbb{R}^{n-k}$ such that

$$
\xi(\tilde{h}(\alpha))=\tilde{S}\left(\begin{array}{c}
\alpha \\
\beta(\alpha)
\end{array}\right)
$$

with some invertible map $\tilde{h}: V \rightarrow U$. 
After the change $x=\tilde{S} \tilde{x}$, system (1) becomes

$$
\tilde{x}^{\prime}(t)=\tilde{S}^{-1} F(t, \tilde{S} \tilde{x}, \varepsilon),
$$

and we have

$$
\tilde{x}(t, z, \varepsilon)=\tilde{S}^{-1} x(t, \tilde{S} z, \varepsilon) .
$$

It is not difficult to see that hypothesis (i) of Theorem 1 of [4] is fulfilled.

The corresponding unperturbed system is

$$
\tilde{x}^{\prime}(t)=\tilde{S}^{-1} F_{0}(t, \tilde{S} \tilde{x}) .
$$

The variational equation of (24) associated to the solution $\tilde{x}(t, z, 0)$ is

$$
y^{\prime}=\tilde{S}^{-1} D_{x} F_{0}(t, \tilde{S} \tilde{x}(t, z, 0)) \tilde{S} y .
$$

Whenever $Y(t, z)$ is a fundamental matrix solution of (14) we have that

$$
\tilde{Y}(t, z)=\tilde{S}^{-1} Y(t, \tilde{S} z)
$$

is a fundamental matrix solution of (25) and, moreover

$$
\tilde{Y}(t, z)^{-1}=Y(t, \tilde{S} z)^{-1} \tilde{S} .
$$

Thus

$$
\tilde{Y}(0, z)^{-1}-\tilde{Y}(T, z)^{-1}=\left[Y(0, \tilde{S} z)^{-1}-Y(T, \tilde{S} z)^{-1}\right] \tilde{S} .
$$

Fix now $Y(t, \xi(h))$ as in the statement of this theorem. Then, from Lemma 3, (21) and (26) written for $z=(\alpha, \beta(\alpha))$, we deduce that hypothesis (ii) of Theorem 1 of [4] is also fulfilled. One can validate by direct and easy calculations the expressions of the bifurcation functions.

\section{Application to the perturbed symmetric Euler top}

We illustrate here our main result Theorem 4 constructing a second order bifurcation function for the perturbed symmetric Euler top. More exactly, we consider system (1) in the case that its dimension $n=3, T>0$ arbitrary but fixed, and the unperturbed system is the symmetric Euler top

$$
\dot{x}_{1}=-x_{2} x_{3}, \dot{x}_{2}=x_{1} x_{3}, \dot{x}_{3}=0,
$$

i.e. $F_{0}(x)=\left(-x_{2} x_{3}, x_{1} x_{3}, 0\right)^{*}$ for any $x=\left(x_{1}, x_{2}, x_{3}\right)^{*} \in \mathbb{R}^{3}$. In [3] it was found that system (27) has the following $T$-period manifolds

$$
\begin{gathered}
\mathcal{Z}_{m}^{v}=\{(0,0, h): h \in(2 m \pi / T, 2(m+1) \pi / T)\} \quad \text { and } \\
\mathcal{Z}_{m}^{h}=\left\{\left(z_{1}, z_{2}, 2 m \pi / T\right):\left(z_{1}, z_{2}\right) \in \mathbb{R}^{2} \backslash\{(0,0)\}\right\},
\end{gathered}
$$

for any $m \in \mathbb{Z}$. Moreover, in [3] it was shown that each of them is normally nondegenerate and we found the expression of the first order bifurcation function for each $T$-period manifold. Here we find the expression of the second order bifurcation function only for the "vertical" $T$-period manifold $\mathcal{Z}_{m}^{v}(m \in \mathbb{Z})$.

We start now checking the hypotheses of Theorem 4 . It is easy to see that $\mathcal{Z}_{m}^{v}$ is the image of $\xi_{m}:(2 m \pi / T, 2(m+1) \pi / T) \rightarrow \mathbb{R}, \xi_{m}(h)=(0,0, h)$ and that the solutions of (27) that initiates in $\mathcal{Z}_{m}^{v}$ are constant (hence trivially periodic). For completeness we prove here that $\mathcal{Z}_{m}^{v}$ is normally nondegenerate. Note that $k=1$. The first variational equations of (27) around the equilibrium $(0,0, h) \in \mathcal{Z}_{m}^{v}$ are

$$
\dot{y_{1}}=-h y_{2}, \quad \dot{y_{2}}=h y_{1}, \quad \dot{y_{3}}=0,
$$


and, moreover, it has the principal fundamental matrix solution

$$
\Phi(t, h)=\left(\begin{array}{ccc}
\cos (h t) & -\sin (h t) & 0 \\
\sin (h t) & \cos (h t) & 0 \\
0 & 0 & 1
\end{array}\right) .
$$

It is easy to check that the kernel of $\Phi(T, h)-I_{3}$ has dimension $k=1$, meaning that the eigenvalue +1 of $\Phi(T, h)$ has geometric multiplicity $k=1$. This shows that the $T$-period manifold $\mathcal{Z}_{m}^{v}$ is normally nondegenerate.

We proceed now to identify all the necessary ingredients to construct $f_{2}$.

The fundamental matrix solution $Y(t, h)$ of $(27)$ can be found following the procedure described in the proof of Lemma 3,

$$
Y(t, h)^{-1}=\left(\begin{array}{ccc}
0 & 0 & 1 \\
\cos (h t) & \sin (h t) & 0 \\
-\sin (h t) & \cos (h t) & 0
\end{array}\right)
$$

and the corresponding matrix (that does not depend on $h$ )

$$
S=\left(\begin{array}{ll}
1 & 0 \\
0 & 1 \\
0 & 0
\end{array}\right)
$$

In order to find the expressions of $g_{0}, g_{1}, g_{2}$ we need the solution of (27) with $x(0)=z=\left(z_{1}, z_{2}, z_{3}\right) \in \mathbb{R}^{3}$,

$$
x(t, z, 0)=\left(\begin{array}{c}
z_{1} \cos \left(z_{3} t\right)-z_{2} \sin \left(z_{3} t\right) \\
z_{1} \sin \left(z_{3} t\right)+z_{2} \cos \left(z_{3} t\right) \\
z_{3}
\end{array}\right) .
$$

We remind that $x(t, \xi(h), 0)=\xi(h)=(0,0, h)$. One can easily obtain

and further

$$
g_{0}(z)=\left(\begin{array}{c}
0 \\
z_{1}-z_{1} \cos \left(z_{3} T\right)-z_{2} \sin \left(z_{3} T\right) \\
z_{2}+z_{1} \sin \left(z_{3} T\right)-z_{2} \cos \left(z_{3} T\right)
\end{array}\right)
$$

$$
\begin{gathered}
\pi g_{0}(z) \equiv 0 \\
-\left[D\left(\pi^{\perp} g_{0}\right)(\xi(h)) S\right]^{-1}=-\frac{1}{2}\left(\begin{array}{cc}
1 & \frac{\sin (h T)}{1-\cos (h T)} \\
-\frac{\sin (h T)}{1-\cos (h T)} & 1
\end{array}\right) .
\end{gathered}
$$

The function $g_{0}$ depends only on the unperturbed system, but $g_{1}$ and $g_{2}$ depend also on the perturbation. Denote the components of the first order (respectively second) order perturbation by $F_{1}=\left(p_{1}, q_{1}, s_{1}\right)^{*}$ (respectively, $\left.F_{2}=\left(p_{2}, q_{2}, s_{2}\right)^{*}\right)$. We are ready now to find that

$$
D\left(\pi g_{1}\right)(\xi(h)) S=\int_{0}^{T}\left(\frac{\partial s_{1}}{\partial x_{1}}(t, \xi(h)), \frac{\partial s_{1}}{\partial x_{2}}(t, \xi(h))\right)\left(\begin{array}{rr}
\cos (h t) & -\sin (h t) \\
\sin (h t) & \cos (h t)
\end{array}\right) d t
$$


By simply calculating the derivatives of $F_{0}$ we obtain that

$$
\sum_{i=1}^{3} \frac{\partial x_{i}}{\partial \varepsilon} \frac{\partial}{\partial x_{i}}\left(D_{x} F_{0}\right) \frac{\partial x}{\partial \varepsilon}=-2\left(\frac{\partial x_{3}}{\partial \varepsilon} \cdot \frac{\partial x_{2}}{\partial \varepsilon}, \frac{\partial x_{3}}{\partial \varepsilon} \cdot \frac{\partial x_{1}}{\partial \varepsilon}, 0\right)^{*} .
$$

Due to the structure of $Y(t, h)^{-1}$, to calculate the expression of $\pi g_{2}$ we need only the third component of $F_{*}$. This implies that the previous sum does not have any contribution to $\pi g_{2}$. In fact finally we have

$$
\pi g_{2}(\xi(h))=\int_{0}^{T}\left(s_{2}(t, \xi(h))+D_{x} s_{1}(t, \xi(h)) \frac{\partial x}{\partial \varepsilon}(t, \xi(h), 0)\right) d t
$$

where

$$
\frac{\partial x}{\partial \varepsilon}(t, \xi(h), 0)=\int_{0}^{t} \Phi(t-\tau, h) F_{1}(\tau, \xi(h)) d \tau,
$$

with $\Phi$ given by (29). The first term in the expression (16) of $f_{2}$ (in fact $f_{2} / 2$ ) is (33), while the second term is the product between (32), (30) and (31). The third term is null since $\pi g_{0}(z) \equiv 0$. We remind that the expression (15) of $f_{1}$ is

$$
f_{1}(h)=\int_{0}^{T} s_{1}(t, \xi(h)) d t
$$

In conclusion, we found in this section the expression of the second order bifurcation function for the problem of persistence of the nonisolated equilibria $\xi(h)=(0,0, h)$, $h \in \mathbb{R} \backslash\{2 m \pi / T: m \in \mathbb{Z}\}$ of the symmetric Euler top (27) as the $T$-periodic solution of the $T$-periodic (sufficiently smooth) system

$$
\begin{aligned}
& \dot{x}_{1}=-x_{2} x_{3}+\varepsilon p_{1}(t, x)+\varepsilon^{2} p_{2}(t, x), \\
& \dot{x}_{2}=x_{1} x_{3}+\varepsilon q_{1}(t, x)+\varepsilon^{2} q_{2}(t, x), \\
& \dot{x}_{3}=\varepsilon s_{1}(t, x)+\varepsilon^{2} s_{2}(t, x) .
\end{aligned}
$$

\section{ACKNOWLEDGEMENTS}

The first and second authors are partially supported by the MICINN/FEDER grant number MTM2011-22877 and by a AGAUR (Generalitat de Catalunya) grant number 2009SGR-381. The first author was also partially supported by a grant of the Romanian National Authority for Scientific Research, CNCS UEFISCDI, project number PN-II-ID-PCE-2011-3-0094. The third author is partially supported by the MICINN/FEDER grant MTM2008-03437, by AGAUR grant number 2009SGR-410 and ICREA Academia.

\section{REFERENCES}

[1] H. Amann, Ordinary differential equations. An introduction to nonlinear analysis, de Gruyter Studies in Mathematics, 13 Walter de Gruyter and Co., Berlin, 1990.

[2] A. Buich̆, J.-P. Françoise And J. Llibre, Periodic solutions of nonlinear periodic differential systems, Comm. Pure Appl. Anal. 6 (2007), 103-111.

[3] A. Buică, I. A. García, Periodic solutions of perturbed symmetric Euler top, Topol. Methods Nonlinear Anal. 36 (2010), 91-100.

[4] A. Buică, J. Giné AND J. Llibre, A second order analysis of the periodic solutions for nonlinear periodic differential systems with a small parameter, Physica D 241 (2012), 528533.

[5] A. BuiC̆ AND J. LliBRe, Averaging methods for finding periodic orbits via Brouwer degree, Bull. Sci. Math. 128 (2004), 7-22.

[6] A. Buic̆ periodic solutions in Lipschitz systems, J. Differential Equations 252 (2012), 3899-3919. 
A. BUICĂ, J. GINÉ AND J. LLIBRE

[7] C. Chicone, Lyapunov-Schmidt reduction and Melnikov integrals for bifurcation of periodic solutions in coupled oscillators, J. Differential Equations 112 (1994), 407-447.

[8] B. Coll, A. Gasull and R. Prohens, Periodic orbits for perturbed non autonomous differential equations, Bull. Sci. Math., in press, http://dx.doi.org/10.1016/j.bulsci.2012.03.001.

[9] J.-P. Françoise, Oscillations en biologie: Analyse qualitative et modèles, Collection: Mathématiques et Applications 46, Springer Verlag, 2005.

[10] I.A. García And J. Giné, The center problem via averaging method, J. Math. Anal. Appl. 351 (2009), no. 1, 334-339.

[11] J. LliBRE, Averaging theory and limit cycles for quadratic systems, Radovi Mat. 11 (2002), $1-14$.

[12] I.G. Malkin, On Poincaré's theory of periodic solutions, Akad. Nauk SSSR. Prikl. Mat. Meh. 13 (1949), 633-646 (in Russian).

[13] M.B.H. Rhouma And C. Chicone, On the continuation of periodic orbits, Methods Appl. Anal. 7 (2000), 85-104.

[14] M. Roseau, Vibrations non linéaires et théorie de la stabilité, (French) Springer Tracts in Natural Philosophy, Vol.8 Springer-Verlag, Berlin-New York, 1966.

Department of Mathematics, Babeş-Bolyai University, RO-400084 Cluj-Napoca, RoMANIA

E-mail address: abuica@math.ubbcluj.ro

Departament de Matemàtica, Universitat de Lleida,, Av. Jaume II, 69, 25001 Lleida, Catalonia, Spain

E-mail address: gine@matematica.udl.cat

Departament de Matemàtiques, Universitat Autònoma de Barcelona, 08193 Bellaterra, Barcelona, Catalonia, Spain

E-mail address: jllibre@mat.uab.cat 